\title{
Cultivation of Data Literacy of University Students in the Era of Big Data: Contents and Countermeasures — Taking Consumer Behaviour Course Teaching as an Example
}

\author{
Qiu-Hong ZHANG
}

College of Economy and Management, Jilin Agriculture University, Changchun,China

Keywords: data literacy; consumer behaviour; course teaching; university students; culture countermeasures

\begin{abstract}
This paper used qualitative research method, depended on teaching characters, studied how to cultivate data literature of university students through $<$ Consumer Behaviour $>$ course. Firstly, based on the researches of predecessors, determined the concept and the associated concepts of the data literacy, and analysed the reasons why the concept of data literacy was put forward. Secondly, it debated the core contents of data literacy in $<$ Consumer Behaviour $>$ course teaching. This paper thought they should include data awareness, abilities of data collection, data handle and management, data analysis and comprehension, decision based on data, and legal and ethical awareness on using data etc. Two sides of awareness and abilities were concluded in the core contents. The lastly, this paper presented countermeasures on cultivation and training the data literacy of university students in $<$ Consumer Behaviour $>$ course teaching. It concluded that should combine the teaching content with the cultivation of data literacy organically.
\end{abstract}

\section{大数据时代大学生数据素养的培养：内容与策略 \\ 一以《消费者行为学》课程教学为例}

\author{
张秋红 \\ 吉林农业大学经济管理学院, 长春, 中国
}

关键词：数据素养；消费者行为学；课程教学；大学生；培养策略

摘要: 本文采用定性研究方法, 根据《消费者行为学》课程教学的特点, 研究了如何通过该 课程教学来培养大学生的数据素养。首先, 在前人研究的基础上, 进一步明确了数据素养的 内涵及其相关概念, 并重点分析了提出数据素养概念的原因。其次, 研究了在《消费者行为 学》课程教学中, 对数据素养培养的核心内容。本文认为, 数据意识; 数据的查找、获取能 力; 加工、处理与管理能力; 分析、理解能力; 使用能力以及使用中的法律、道德意识等内 容是目前课程教学中对大学生数据素养培养的主要内容。数据素养的核心内容可分为思想意 识和行为能力两个层面, 最后提出了在《消费者行为学》课堂教学中, 培养大学生数据素养 的策略。得出结论，应该将教学内容与数据素养培养有机结合。

\section{1. 引言}

在大数据时代，数据已经逐渐成为政府、企业以及个人进行决策的依据和创新的驱动力 量。大学生作为思想、认知最为活跃的一个群体，也是目前大数据的主要创造者之一，必将 成为未来大数据分析和使用的重要人群。在大学教育阶段, 积极培养大学生的数据素养, 增 强大学生对数据价值的认知, 以及利用数据分析解决现实问题和驱动创新的能力, 无论对于 个人还是对于国家，都必将是一项非常具有现实价值和长远意义的工作。《消费者行为学》是 
一门研究消费者心理特征与行为规律的科学, 过去常采用随机抽样调研的方法开展研究。随 着大数据时代的到来, 人们获取、使用数据日益便捷, 越来越多的研究者开始采用能更好反 映消费者实际情况的大数据开展研究。而本科教育培养的是日后的研究者和实践者, 学生们 未来走上工作岗位, 必然要面临大数据的提供、处理与分析。因此, 目前的课堂教学中加强 学生们数据素养的培养势在必行。

\section{2. 数据素养的内涵及其被提出的意义}

\section{1 数据素养的内涵}

素养 (literacy) 一词的英文原意是 “识文断字、能读能写”。汉语字典中的解释为: 基本 的素质与修养。可见, 无论中西方语境, 素养都是指 “最基本的素质和能力”。那么, 数据素 养就应该是人们对于数据的最基本的素质和能力。因此, 可以将数据素养定义为: 获取、解 释、批判性的评价、管理、处理和有效使用数据, 并最终将数据转化为知识的能力, 以及在 此过程中的道德和行为规范。(J.C.Prado and M.A.Marzal, 2013; wikipedia, 2013; 张静波, 2013 ) 在大数据时代, 不仅需要具有数学和计算机学科背景的专业数据分析师, 同时需要更 多的是具有不同学科背景且能够依据大数据研究进行相应决策的各类人才。

\section{2 提出数据素养的意义}

数据素养这一概念的提出意义深远：首先，大数据时代的数据与传统意义的数据有显著 分别, 导致从数据中提取有价值的信息变得更难。数据是以数字、文字、图像、视频、音频 或计算机代码等形式存在的一组物理符号。数据本身并无价值, 通过对数据的分析与解读从 而获得信息才有价值。因此, 数据就是信息的载体, 人们要想获得信息, 就先要对数据进行 处理、分析和解读。在过去, 人们生成和存储的数据量十分有限, 且它们多半以数值的形式 存在。因此对数据进行一定的处理和分析, 就可以获得有价值的信息。但是, 大数据时代的 数据具有体量庞大 (Volumn)、模态繁多 (Variety)、生成快速 (Velocity) 和价值巨大但低密 度（Value）的特征。（李国杰，2012）例如, 现在每两天就可以创造出自人类文明诞生以来 到 2003 年的数据量, 且其中 $75 \%$ 都以日志、图像、视频、音频等半结构化或非结构化的形式 存在, 想要从中获得真正有价值的信息变得不是更容易而是更难。因此, 在大数据时代, 如 果只关注 “能否认识到信息的价值? 会不会搜索信息? 会不会使用 “百度” 等搜索引擎? 会 不会使用 “Excel' 等信息处理软件” (1)等与信息素养有关的基础问题显然不够, 更跟不上时 代的发展。大数据时代的真正挑战在于 “如何看待生活中每时每刻生成的数据? 如何处理这 些数据？如何从中获取有价值的信息？”，而这些都是与数据素养紧密相关的问题。

其次，伴随着互联网与移动互联网的迅速发展和广泛应用，使用计算机和互联网进行日 常信息处理, 已经成为大学生群体几乎人人具备的一项基本技能。在获取信息的平台制约变 得越来越小的情况下, 关注信息的载体一一数据的认知、理解、分析和使用的能力就显得更 加重要。现在, 无论是传统行业还是新兴行业, 亦或是政府部门, 在进行决策时, 数据都在 扮演着越来越重要的角色。即使是普通百姓的日常生活决策, 也在受着数据的影响。例如, 百姓通过对物价指数、股票指数、空气污染指数等数据的解读来感受自己所生活的社会、经 济、环境的状况, 从而作出个人决策。(金兼斌, 2013) 在电脑、互联网、移动互联工具逐渐 普及的情况下, 认知、理解数据的价值和局限性, 并用好数据, 成为一个有数据素养的人, 关乎一个社会的整体理性水平, 也将是下一阶段信息素养竞争与培养的关键。(缪其浩, 2013b)

\footnotetext{
(1) 这些问题也是目前国内学者在研究大学生、青少年信息素养时经常提出和调研的问题。具体可参见刘航等《信息化时代大 学生信息素养现状及培养对策》, 《情报科学》2013 (11); 路强等《大学生信息素养现状分析与培养途径研究》, 《情报科学》 2013 (10); 兰亚春等《网络时代青少年信息素养现状及其培养途径》, 《情报科学》2013 (9); 李娅等《高校学生信息素养 现状及其评价标准研究进展》, 《农业图书情报学报》, 2013 (2) 等。
} 


\section{3 《消费者行为学》课程教学中数据素养培养的核心内容}

数据素养是一个包含多个维度的综合性概念。它具体应该包括如下方面:

第一，数据意识。具体指能够认识到数据是有价值的; 对与自己工作、生活、学习相关 的数据具有敏感性, 并有获取这些数据的兴趣; 能够意识到数据在社会中扮演的角色, 以及 数据对科学研究和社会的影响; 理解数据是如何产生的, 是由哪些人或者哪个部门生成的; 这些数据的可能应用领域、使用权力和时效等; 能够认知到个人在网络及现实生活中可能留 下的 “数据痕迹”, 以及个人隐私的暴露程度等。

第二, 数据的查找、获取能力。具体包括知道获取数据的可能来源与渠道, 并能对这些 来源与渠道的权威性、可靠性等加以评估, 并从中加以选择; 能明确现有数据对现有问题的 解决程度, 并能为解决问题进一步搜索新的数据。

第三, 数据的加工、处理与管理的能力。具体指能够对所获得的数据进一步加工和处理, 会使用常用的数据处理工具 (SPSS、Excel 等), 并能够根据个人使用的需要和目标对数据进 行重新的合成与再现; 能够有意识的存储、保护已经获得或生成的数据, 以便未来可以进一 步使用; 会使用常用的线上、线下数据存储工具。

第四, 数据的分析、理解能力。具体指能意识到数据可能表现的各种各样形式 (不仅表 现为数值, 还可以表现为文本、图像、音视频等), 并能理解每种数据形式所代表的真正含义; 能够对各种形式的数据加以分析、解释; 能够对数据本身加以批判性的评价; 理解数据在生 成、获取、分析时所采用方法的局限性; 理解数据中可能存在的误差以及数据本身解决问题 的局限性。

第五，使用数据解决现实问题或进行决策的能力。具体指能够应用数据帮助解决工作、 生活或学习中遇到的实际问题, 或者帮助进行相关决策, 而不是仅仅凭借个人直觉进行决策; 能够对数据解决问题的程度和局限性加以评估; 当用数据作为决策的依据时，可以用合适的 方式对数据加以呈现，从而让他人更好地理解和接受。

第六，在使用数据的过程中具备所需的法律、道德意识。具体指能够认知到在数据的搜 集、处理和使用过程中所产生的经济、社会与法律问题; 在获取、使用数据时遵守法律和社 会伦理道德; 理解数据的使用权限, 保护个人隐私; 对外公布的相关数据和结果应真实有效。

\section{4 《消费者行为学》课程教学中培养学生数据素养的策略}

数据素养的核心内容可以归纳为两个层面：思想意识层面和行为能力层面。对于这两个 层面的培养，采取的策略不尽相同。培养思想意识最有效的方法是环境的塑造。“蓬生麻中， 不扶自直”。如果在大学生学习、生活的环境中, 绝大多数人都具有很强的数据意识, 深知数 据的作用和价值, 常用数据并明确相应的法律、道德问题, 那么, 大学生自身也会受到环境 的影响，逐渐形成数据意识。但对于行为能力的培养则不同。行为能力不同于一般知识，后 者只要在课堂中讲授, 在头脑中形成记忆就可以称为掌握; 但前者必须通过不断的练习和实 际应用才能够被掌握; 同时对于行为能力的培养也没有 “终点”, 需要随着环境的变化而不断 调整、巩固和提高。因此，培养大学生的数据素养，可以有针对性地采取以下策略:

首先，营造良好的数据素养培养环境。《消费者行为学》课程虽不如计算机、数学、统计 学等课程属于直接涉及数据的课程, 但后面的课程主要以数据处理为主, 而前者则以数据应 用为主。教师在日常授课时, 应有意识的主动使用数据来讲述课程内容, 佐证自己的观点和 结论。在 2012 年伦敦奥运会的报道中, 英国《卫报》的 “数据博客” 大放异彩, 吸引了众多 网友和观众。在《消费者行为学》的讲授中, 教师也可以效仿现在新闻媒体对数据的应用, 将数据渗透到课堂, 例如, 在讲授消费者的动机、态度时, 将最新的调研数据结果渗入, 这 样不仅可以让学生有机会更多的接触数据, 认识到数据的意义和价值, 还可以让学生更进一 步的学会如何分析、使用数据。 
其次, 将数据素养的培养与课程的教学整合在一起。根据国外的经验, 将数据素养的相 关知识与技能的培养嵌入到各个专业课程的教学中, 比单独开设相关的数据素养课程的效果 要好。(E.Stepheson and P.S.Caravello, 2007) 因为, 通过这样的嵌入与整合, 学生可以在一 般课程的学习中不断接触到数据, 这对于提升他们的数据敏感性和对数据价值的认知非常有 帮助。同时, 专业课程的学习如果能够经常要求学生搜集数据, 处理和分析数据, 使学生不 断有机会练习和使用上述技能, 这对于培养和提升他们的数据获取、处理、分析的能力效果 非常好。因此, 《消费者行为学》课程也应该学习国外经验, 努力将数据素养的培养与课程的 讲授整合在一起。

最后, 重点关注对大学生的数据分析、使用能力的培养。在大数据时代, 需要处理的是 体量巨大、类型丰富的, 多数是半结构化或非结构化的数据, 传统的数据处理方法难以应对, 需要开发新的方法与工具。因此, 在这种背景下, 数据素养的培养很容易被引入到技术层面, 即关注怎样开发出适合处理 “大数据” 的新工具, 如何使用这些工具等。但是, 数据处理只 是手段, 根本目标是从数据中挖掘出有价值的信息, 并帮助人们决策。所以, 在大数据时代, 数据的分析、使用能力更加重要。正如 Jeanie Harris（2012）所言 “如果没有分析能力, 数据 本身毫无意义。” 因此, 高校在培养大学生数据素养时, 不仅要关注对学生数据获取、处理、 管理能力的培养, 更应该注重对学生的数据分析、使用能力的培养。即培养学生深入分析问 题的能力以及质疑的思想, 不仅要对数据具有敏感性, 更要理解数据背后的真正意义, 不被 所谓的 “精确数据” 所左右。《消费者我希望》正式一门很好的数据应用的课程, 可以通过课 程作业、实验等方式锻炼学生数据分析、处理、使用的能力。

\section{致谢}

本文为吉林农业大学高等教育教学研究课题《<消费者行为学>教学内容与教学方法改革 研究》(2017XJZD09); 吉林农业大学博士启动基金项目《农产品品牌真实性对在线消费者购 买行为的影响研究》(201640) 的阶段性成果之一。

\section{References}

[1] Jing-Bo ZHANG.The education ofData literacy in big data era, Science, vol 65,pp.29-32,2015.

[2] J.C.Prado, M.A.Marzal. Incorporating Data Literacy into Information Literacy Programs: Core Competencies and Contents, Libri, vol63, pp123-134,2013.

[3] Wikipedia.Dataliteracy, http://en.wikipedia.org/wiki/Data-literacy.

[4] Guo-Jie LI. Big data research: a major strategic field of future science, technology and economic and social development - research status and scientific thinking of big data, Bulletin of the Chinese Academy of Sciences, vol27, pp 647-657,2013.

[5] Jeanie Harris. Data is Useless Without the Skills to Analyze It, http://blogs. Harvard Business Review.org/2012/09/13/. 